\title{
Flow field and flame surface density of turbulent flames by simultaneous PIV/OH-PLIF measurements
}

\author{
Kazuhiro YAMAMOTO*, Kenta YAMAMORI* and Yasuo ASADA* \\ * Department of Mechanical System Engineering \\ Nagoya University, Furo-cho, Chikusa-ku, Nagoya, Aichi 464-8603, Japan \\ E-mail: kazuhiro@mech.nagoya-u.ac.jp
}

Received: 9 October 2017; Revised: 13 December 2017; Accepted: 22 February 2018

\begin{abstract}
In this study, we have examined the flow field and the flame surface density in premixed combustion by simultaneous particle image velocimetry (PIV) and OH-planar laser induced fluorescence (OH-PIIF) measurements. Turbulent flames are formed in a cyclone-jet combustor under weak to highly turbulence. The flame surface density is evaluated by the orthogonal OH-PLIF for vertical and horizontal imaging. In terms of the flame front detected by $\mathrm{OH}$ fluorescence, the flow field of turbulent flames including the strain rate has been discussed. It is found that the axial velocity becomes the maximum around the center axis, whereas the rms axial or radial velocity takes its maximum at the position far from the center axis. The larger velocity fluctuation is observed by increasing the mean exit velocity of $U_{m}$. The radial position of peak $\mathrm{OH}$ fluorescence signal is not in the region of large rms velocity, except for the condition where the local extinction is observed. Hence, the large velocity fluctuation induces the local extinction through the high strain rate. The flame surface density is increased with an increase in rms velocity. The range of three-dimensional flame surface density (3D FSD) is from 0.2 to $0.8 \mathrm{~mm}^{-1}$, which corresponds similarly to that of the Bunsen flame. Moreover, based on two-dimensional (2D) and 3D FSDs, the increasing factor of 3D effects is not the same value of homogeneous turbulence. Recognizing a linear relationship between the rms axial velocity and the integrated FSD, the increase of FSD is simply caused by the flame wrinkling due to the turbulence, even at the condition where the local extinction occurs.
\end{abstract}

Keywords : Turbulent combustion, Premixed combustion, OH-PLIF, PIV, Strain rate

\section{Introduction}

So far, many researches have been conducted for modeling turbulent combustion including theoretical, experimental and numerical studies [Peter, 1986, 1999, Nishiki et al., 2002, Bradley et al., 2003]. Based on the outcomes of these studies, it has been proposed that several different regimes are given for classifying the turbulent flame structure. In the most recent regime diagram for premixed turbulent combustion, the traditional flamelet regime has been extended further up to $K a=100$ from the previous upper limit of $K a=1$, where Karlovitz number $(K a)$ is defined as the ratio of chemical time scale to the Kolmogorov time scale [Gülder, 2007]. Recently, by the drastic improvement of laser diagnostics, it has been possible to validate the proposed turbulent models in terms of instantaneous flame images [Osborne et al., 2017, Wabel et al., 2017]. The region between $K a=1$ and $K a=100$ is called the thin reaction zones regime, and the flamelet assumptions are still valid. In our work [Yamamoto et al., 2011], it is concluded that the turbulent burning velocity $\left(S_{T}\right)$ is well described by the Damköhler's formula and the bending in $S_{T}$ could be caused by the suppression of the local burning velocity. Within the frame work in our previous study, the flamelet approach is still applicable.

Apparently, there are several factors for characterizing the turbulent premixed flames [Filatyev et al., 2005]. Since $S_{T}$ is increased by the enlargement of the flame front surface [Kido et al., 2002, Gülder, 2007, Chen, 2009], the flame surface density (FSD) could be an important parameter which can quantify the turbulent flames. In a series of studies, the model with FSD transport equations has been tested to predict turbulent premixed flames [Cant, et al., 1990, Filatyev et al., 2005, Driscoll, 2008, Hawkes et al., 2011]. For example, it is found that, under the weak turbulent flow, 
the local perturbation of flamelet structure affected by turbulent eddies is well describe by FSD [Jainski et al., 2017]. However, it is quite difficult to evaluate the flame structure based on the three-dimensional (3D) measurement. Most of studies were performed by two-dimensional measurements such as PLIF techniques. Uniquely, some advanced approaches have been proposed [Chen, 2009, Yamamoto et al., 2011, Hawkes et al., 2011]. However, less data on FSD is available because of difficulties in measurements. Especially, the effect of local extinction is not clear.

In this study, we investigated the combustion field of highly turbulent premixed flames by simultaneous particle image velocimetry (PIV) and OH-planar laser induced fluorescence (OH-PIIF) measurements. The local flame structure was visualized by the orthogonal OH-PLIF measurement to visualize the two-dimensional (2D) flame zones in the vertical and the horizontal planes, which gave us the information on FSD of 3D flame structure. The swirling turbulent flames were formed by a cyclone-jet combustor in the wide turbulence range [Yamamoto et al., 2003, 2007, 2009, 2011, 2016, Suzuki et al., 2013]. Then, focusing on the local extinction, we discussed the flame and the flow interactions.

\section{Experimental setup}

\subsection{PLIF system}

The experimental setup for PLIF system is shown in Fig. 1. The orthogonal OH-PLIF measurements were applied to evaluate the 3D flame surface. The vertical plane was obtained by a laser sheet 1 , and the horizontal plane was obtained by a laser sheet 2 . Since the same excitation line was used for both imaging, the time delay of $100 \mathrm{~ns}$ between two laser pulses was set to distinguish these fluorescence signals. Two wavelength-tuned laser beams were expanded to thin laser sheet (its thickness is less than $0.4 \mathrm{~mm}$ ) by spherical lenses and cylindrical lenses, then exposed to the flames. A frequency doubled (532nm, 10Hz) Nd:YAG laser (GCR-230, Spectra Physics Inc.) pumped a Dye laser (HD-300, Lumonics Inc.) whose frequency was doubled to $283.2 \mathrm{~nm}$ to excite the $\mathrm{Q}_{1}(7)$ line of $(1,0)$ band in $A^{2} \Sigma^{+} \leftarrow X^{2} \Pi$ transition, ensuring that the fluorescence signal was less dependent on the temperature [Yamamoto et al., 2009]. The total energy per pulse was about $10 \mathrm{~mJ}$. In our preliminary studies, it has been confirmed that the $\mathrm{OH}$ fluorescence signal was proportional to the molar concentration and the error was within $10 \%$.

For the fluorescence imaging, two intensified CCD cameras (C8484 \& C9164, Hamamatsu Photonics) with an array size of 1000 x 500 pixels were used. An interference filter centered at $307 \mathrm{~nm}$ (12 nm bandwidth) was placed in front of the 105-mm f/4.5 UV-Nikkor lens. Each capture area was $25 \mathrm{~mm}$ x $12.5 \mathrm{~mm}$, and the spatial resolution was 25 $\mu \mathrm{m} /$ pixel. In the horizontal plane, the imaging area was $18.3 \mathrm{~mm} \times 18.3 \mathrm{~mm}$, and the corresponding pixel number was $680 \times 680$. The spatial resolution was about $27 \mu \mathrm{m} /$ pixel. A set of 1000 images was obtained for the statistical analysis.
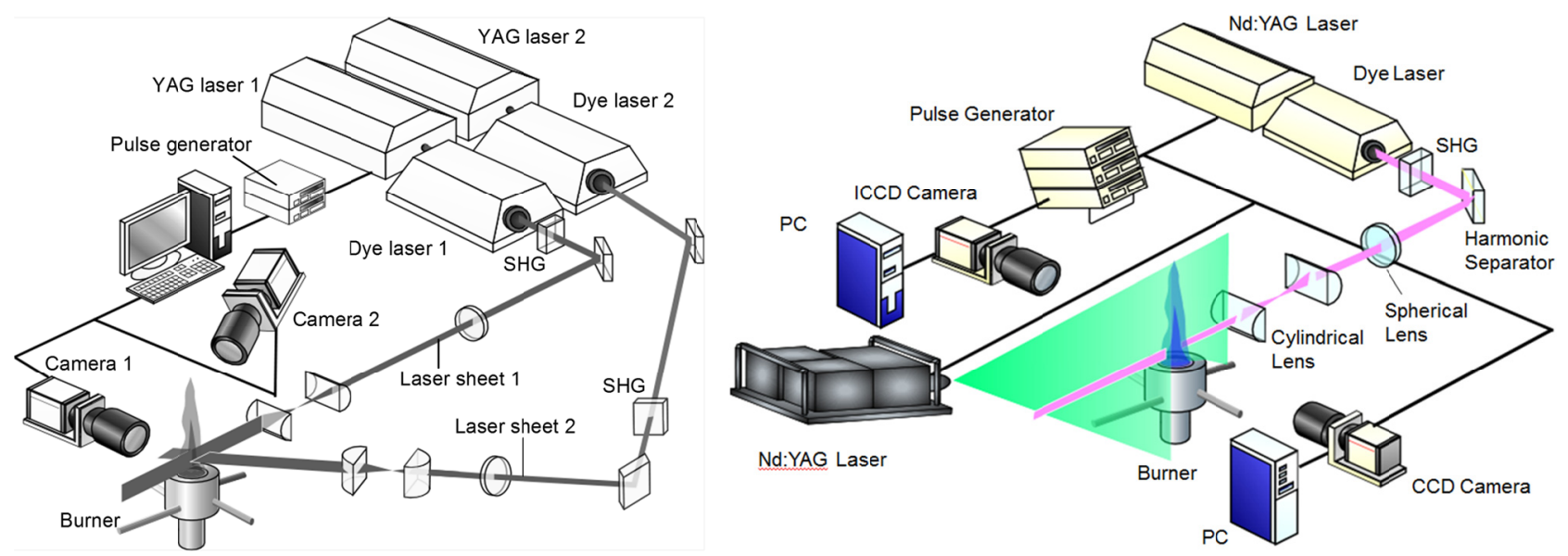

Fig. 1 Left figure shows the orthogonal OH-PLIF in vertical and horizontal planes. Right figure shows the experimental setup for simultaneous PIV/OH-PLIF measurement.

\subsection{PIV system}

To make clear the effect of turbulence on the flow and the flame structure, it would be useful to visualize the 
two-dimensional velocity field by a Particle Image Velocimetry (PIV) technique. In order to evaluate the flame and the flow interactions, the simultaneous vertical PIV/OH-PLIF measurement was conducted. The experimental setup is shown in Fig.1. The PIV system consisted of a Nd:YAG laser $(532 \mathrm{~nm}, 10 \mathrm{~Hz})$ and a CCD camera (TSI PIVCAM13-8). The 532-nm frequency-doubled beam was used to produce a laser sheet of $500 \mu \mathrm{m}$ thickness through the center of the burner. The time between two PIV pulses were remained constant at $10 \mu \mathrm{s}$, and two consecutive raw PIV images were collected on the $1024 \times 1280$ pixel array of the CCD camera. The velocity was evalluated using the standard cross-correlation method, with interrogation window of $16 \times 16$ pixels overlapped by $50 \%$, providing a matrix of 127 $(z) \times 159(r)$ displacement vectors, where $z$ represented the axial distance from the combustor exit and $r$ was the radial axis. The size of the test window and the range of integration were selected based on our previous experiments [Yamamoto et al., 2006]. The axial velocity was $u$, and the radial velocity was $v$ in this study. Resultantly, the spatial resolution was about $0.25 \mathrm{~mm}$. $\mathrm{MgO}$ of $1.8 \mu \mathrm{m}$ mean diameter was used for particle scattering [Yamamoto et al., 2007, 2016, Suzuki et al., 2013]. The background emission was suppressed with the interference filter centered at $532 \mathrm{~nm}$ with a 10-nm bandpass. To confirm the validity of PIV data, an LDV system [Yamamoto et a1., 2007] was also used.

Typically, an imaging area by PIV was at $z=5$ to $35 \mathrm{~mm}$ and $r=-20$ to $20 \mathrm{~mm}$. Avallone et al. (2003) have conducted PIV measurements on the flow with the grid turbulence, where 1000 data are used to discuss the velocity and flame characteristics statistically. Thus, for each experimental condition, we obtained 1000 images of velocity field and OH-PLIF. For comparison, the velocity field of the cold flow (unburned gas) was also measured.

\subsection{Cyclone-jet combustor}

In this experiment, the cyclone-jet combustor (C-J combustor) was used to stabilize the turbulent premixed flames, which is shown in Fig. 2. It consisted of a combustion chamber with a main jet nozzle and four cyclone nozzles for pilot flames. The diameter of the main jet nozzle was $12.7 \mathrm{~mm}$ and that of the cyclone combustor was $21 \mathrm{~mm}$, with four cyclone nozzles of $2.4 \mathrm{~mm}$ i.d. Due to a circular pilot flame inside the combustor, the flame was stabilized at the combustor exit, and hence, it was easy to examine the combustion field by laser diagnostics. In the experiment, we varied the mean exit velocity, $U_{m}$, and the equivalence ratio, $\phi_{m}$, of the main jet, with a fixed condition of pilot flames for $U_{p}=10 \mathrm{~m} / \mathrm{s}$ and $\phi_{p}=0.7$. The fuel was propane.

In our previous measurements by LDV and PIV, the turbulent Reynolds number, $R_{T}$, was from 96 to 448 for $U_{m}=$ 5 to $30 \mathrm{~m} / \mathrm{s}$ and $\phi_{m}=0.75,0.9$ [Yamamoto et al., 2003, 2007]. On the phase diagram, the condition of $U_{m}<15 \mathrm{~m} / \mathrm{s}$ and $\phi_{m}=0.75$ or $U_{m}<20 \mathrm{~m} / \mathrm{s}$ and $\phi_{m}=0.90$ belongs to the flamelet regime, and that of $U_{m}>20 \mathrm{~m} / \mathrm{s}$ and $\phi_{m}=0.75$ or $U_{m}=$ $30 \mathrm{~m} / \mathrm{s}$ and $\phi_{m}=0.90$ belongs to the thin reaction zones regime. The flame was completely extinguished slightly above $30 \mathrm{~m} / \mathrm{s}$ at $\phi_{m}=0.75$, corresponding to the global flame extinction.

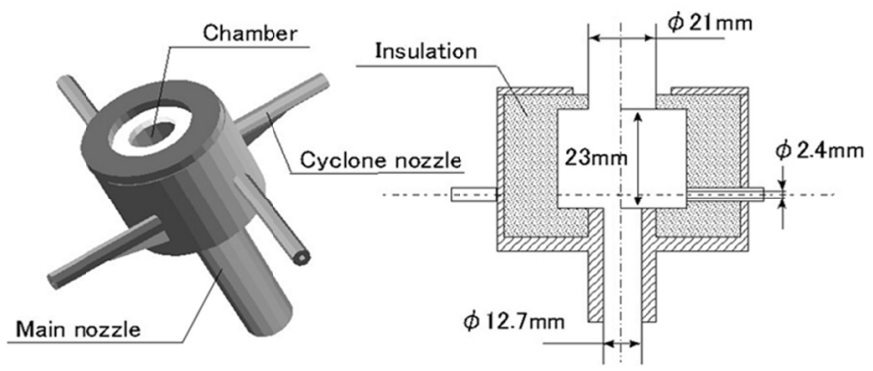

Fig. 2 A schematic of cyclone-jet combustor.

\section{Results and discussion \\ 3.1 Appearance of flame}

First of all, the appearance of the flame in the C-J combustor is shown. Photographs of flames are shown in Fig. 3. The exposure time was $1 / 500 \mathrm{~s}$ and the diaphragm was F2.8. As seen in this figure, it is found that the flame length is longer as the exit velocity is higher. On the other hand, as the equivalence ratio is increased, the flame height is lower. These characteristics are similar to that of a so-called Bunsen flame. For simple calculation, the global stretch rate was 
$9501 / \mathrm{s}$ for $U_{m}=10 \mathrm{~m} / \mathrm{s}$ or $28501 / \mathrm{s}$ for $U_{m}=30 \mathrm{~m} / \mathrm{s}$, which was roughly estimated from the mean exit velocity of $U_{m}$ divided by the radius of the combustor exit $(=10.5 \mathrm{~mm})$.

As discussed later, the local quenching occurred for $U_{m}=30 \mathrm{~m} / \mathrm{s}, \phi_{m}=0.75$. Needless to say, we cannot detect the local extinction based on these figures. This is because the direct photograph only shows the averaged flame shape, and the fast flame motion is not captured. At the next section, we will discuss the instantaneous flame structure.

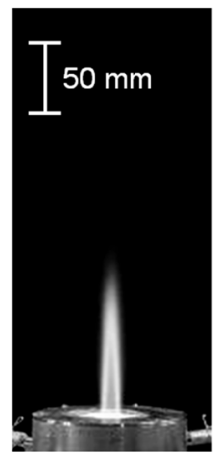

(a)

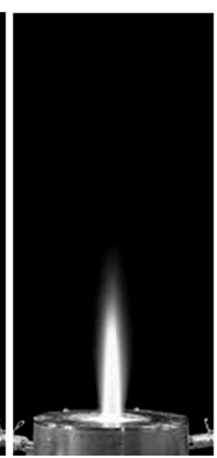

(b)

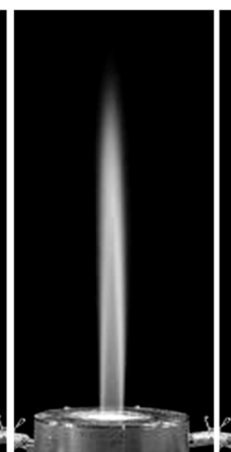

(c)

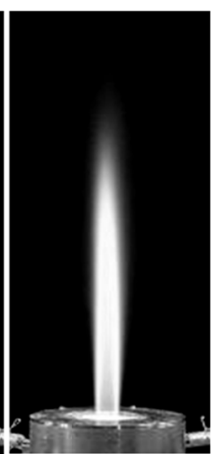

(d)

Fig. 3 Photographs of flame in a cyclone-jet combustor; (a) $U_{m}=10 \mathrm{~m} / \mathrm{s}, \phi_{m}=0.75$, (b) $U_{m}=10 \mathrm{~m} / \mathrm{s}, \phi_{m}=0.90$, (c) $U_{m}=30 \mathrm{~m} / \mathrm{s}, \phi_{m}=0.75$, (d) $U_{m}=30 \mathrm{~m} / \mathrm{s}, \phi_{m}=0.90$.

\subsection{OH image and velocity field}

Examples of the OH-PLIF image and the velocity field are shown in Fig. 4. For $U_{m}=10 \mathrm{~m} / \mathrm{s}, \phi_{m}=0.75$, it is seen that there is one continuous flame front, and the flame is wrinkled by the turbulence. For higher equivalence ratio in Fig. 4b, the flame diameter becomes smaller due to the larger burning velocity. In Fig. 4c, OH region is distorted and collapsed, which is caused by the local extinction [Yamamoto et al., 2009, 2011]. By comparing Figs. 4c and 4d, it is found that, at the higher equivalence ratio, the flame front recognized by $\mathrm{OH}$ fluorescence intensity is more wrinkled.

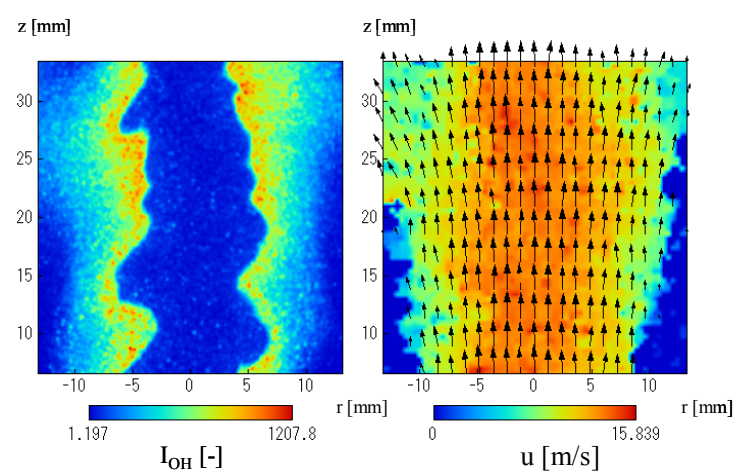

(a) $U_{m}=10 \mathrm{~m} / \mathrm{s}, \phi_{m}=0.75$

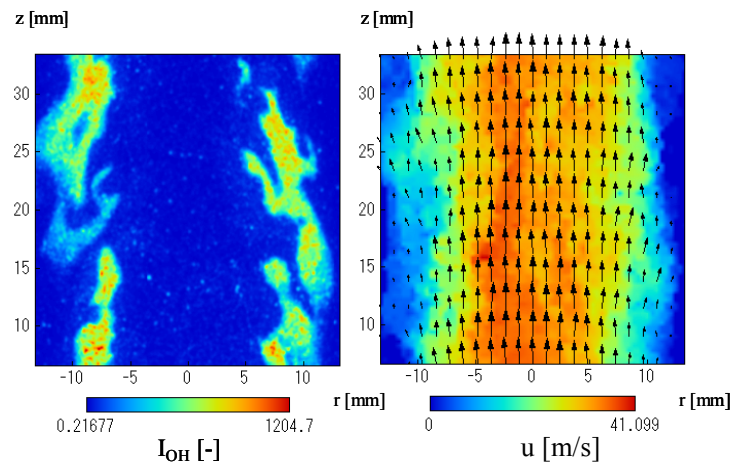

(c) $U_{m}=30 \mathrm{~m} / \mathrm{s}, \phi_{m}=0.75$

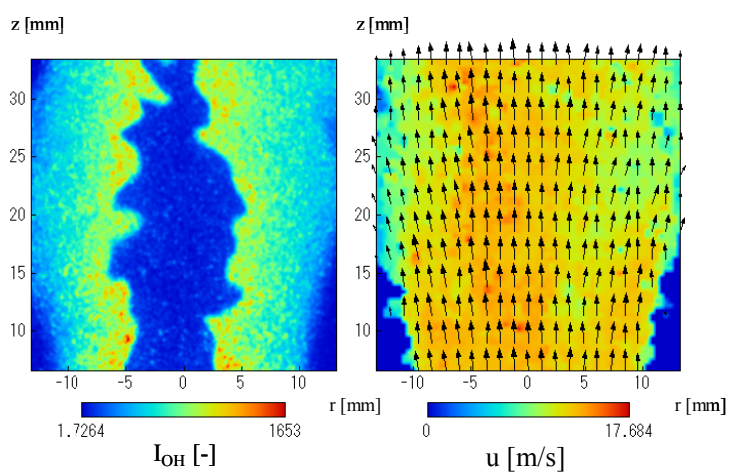

(b) $U_{m}=10 \mathrm{~m} / \mathrm{s}, \phi_{m}=0.90$

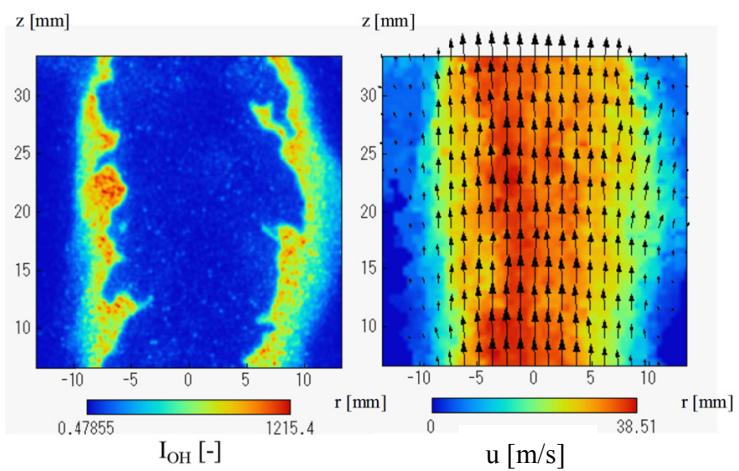

(d) $U_{m}=30 \mathrm{~m} / \mathrm{s}, \phi_{m}=0.90$

Fig. $4 \mathrm{OH}$ image in vertical plane (left) and axial velocity of $\mathrm{u}$ with velocity vector (right). 
Next, the flow field is discussed. Apparently, the jet structure of the cyclone-jet combustor is radially extended further downstream [Yamamoto et al., 2007]. It is noted that, as the mean exit velocity of $U_{m}$ is increased, the measured axial velocity is expectedly higher. To see the flow field in detail, the radial distributions of the axial velocity are shown with $\mathrm{OH}$ fluorescence signals in Fig. 5. The flow conditions are the same in Fig. 4. The axial position of these figures are at $z=25 \mathrm{~mm}$. There are roughly three regions: no $\mathrm{OH}$ (unburned gas), low $\mathrm{OH}$ (burned gas), and high $\mathrm{OH}$ (super-equilibrium $\mathrm{OH}$ in reaction zone). The flame front was determined at the position where $\mathrm{OH}$ became the half value of the peak $\mathrm{OH}$ [Yamamoto et al., 2007]. The axial velocity becomes the maximum around the center axis. When the mean exit velocity is higher, the larger shear flow exists due to the jet flow from the burner exit. In Fig. $5 \mathrm{c}$, the $\mathrm{OH}$ fluorescence signal is almost zero at $7 \mathrm{~mm}<r<10 \mathrm{~mm}$ where the local flame extinction appears, which will be discussed in section 3.3 .

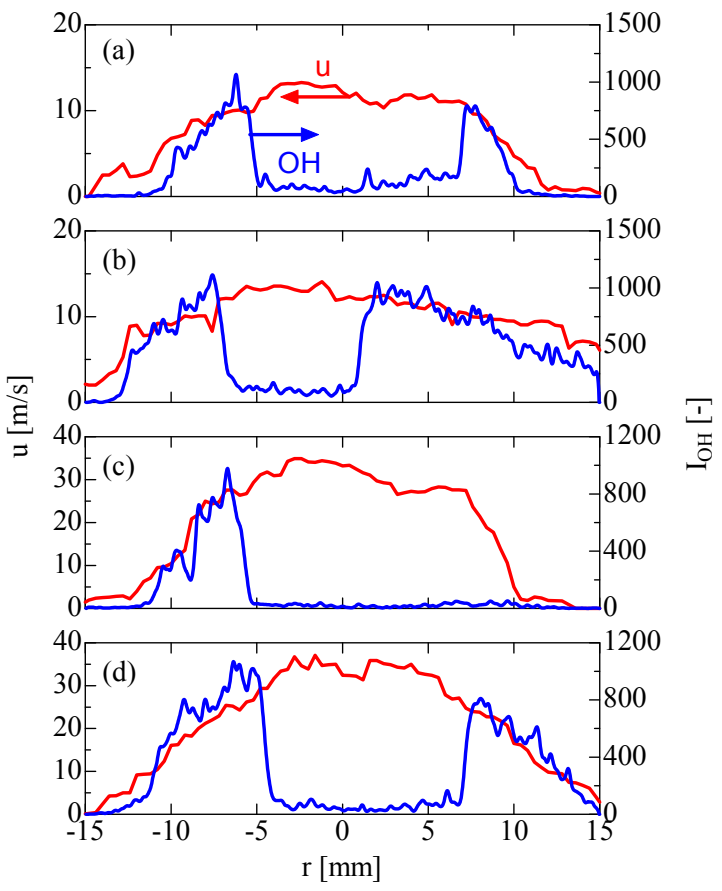

Fig. 5 Radial distributions of $\mathrm{OH}$ fluorescence signal and axial velocity are shown; (a) $U_{m}=10 \mathrm{~m} / \mathrm{s}, \phi_{m}=0.75$, (b) $U_{m}=10$ $\mathrm{m} / \mathrm{s}, \phi_{m}=0.90$, (c) $U_{m}=30 \mathrm{~m} / \mathrm{s}, \phi_{m}=0.75$ and (d) $U_{m}=30 \mathrm{~m} / \mathrm{s}, \phi_{m}=0.90$. These are obtained at $z=25 \mathrm{~mm}$.

\subsection{Statistical analysis of velocity and $\mathrm{OH}$ signal}

For further discussion, we statistically analyzed the velocity data. Figure 6 shows the maximum of axial and radial velocities obtained at $z=15 \mathrm{~mm}$. The flow conditions are $U_{m}=10$ or $30 \mathrm{~m} / \mathrm{s}$, and $\phi_{m}=0.75$ or 0.90 . In this figure, the radial positions where these maximums were measured were specified. To examine the effect of the flames, the measurements of the cold jet were also conducted. To see the difference between four plots, only 100 out of 1000 were randomly chosen and plotted. It is seen that the maximum axial velocity is observed around the center axis. On the other hand, the maximum radial velocity is observed at $r=5 \mathrm{~mm}$ to $14 \mathrm{~mm}$ (or $r=-14 \mathrm{~mm}$ to $-5 \mathrm{~mm}$ ), at which the radial velocity must be accelerated due to the heat expansion. At $U_{m}=10 \mathrm{~m} / \mathrm{s}$ and $\phi_{m}=0.75$, the maximum of the axial velocity $(u)$ is slightly larger than the averaged velocity of $10 \mathrm{~m} / \mathrm{s}$. Some variations in the maximum velocity of $u$ and $v$ are observed in the case of the combustion. Similarly, in the case of the cold flow without flames, the maximums of the axial and radial velocities are varied. However, the radial position where the maximum of the axial velocity is obtained largely varies. These findings correspond to the fact that the velocity decay of the cold jet is much larger, which is well observed in profiles of $u^{\prime}$ and $v^{\prime}$ at the center axis [Yamamoto et al., 2007].

Next, we examine the velocity fluctuation and the $\mathrm{OH}$ signal. Figure 7 shows the radial distributions of rms axial and radial velocities, $u$ ' and $v^{\prime}$, obtained at $z=15 \mathrm{~mm}$. For comparison, the distribution of mean $\mathrm{OH}$ fluorescence signal is plotted. As already mentioned, the axial velocity becomes the maximum around the center axis. Yamamoto et al. (2007) have shown the same tendency observed in the cold jet in the C-J combustor. On the other hand, $u$ ' becomes large at $6 \mathrm{~mm}<r<12 \mathrm{~mm}$ (or $-12 \mathrm{~mm}<r<-6 \mathrm{~mm}$ ), which is far from the center axis. Interestingly, for all cases, the 
profile of $u^{\prime}$ is quite similar to that of $v^{\prime}$. That is, a good correlation between rms axial and radial velocities is observed. Recognizing that the flame is fluctuating at the region of the large shear layer in Figs. 4 and 5, the large velocity fluctuation in Fig. 7 is caused by the flame motion, because there is the flow expansion due to the temperature rise in the combustion field.

Next, the rms velocity and the $\mathrm{OH}$ profile are compared. Except for the condition of $U_{m}=30 \mathrm{~m} / \mathrm{s}$ and $\phi_{m}$ in Fig. 7c, the radial distance of the peak $\mathrm{OH}$ fluorescence signal is slightly inside the peak position of $u$ ' and $v$ '. As mentioned before, the large velocity fluctuations are due to the presence of the flame. However, for the condition of $U_{m}=30 \mathrm{~m} / \mathrm{s}$ and $\phi_{m}$ in Figs. $4 \mathrm{c}$ and 5c, the local extinction is observed, resulting in the large reduction of OH. Indeed, the averaged $\mathrm{OH}$ signal is much lower than those of other three cases. Expectedly, at the region of the local extinction, the velocity may decrease due to the reduction of temperature, but by contrast, the values of $u^{\prime}$ and $v^{\prime}$ at $6 \mathrm{~mm}<r<9 \mathrm{~mm}$ (or -9 $\mathrm{mm}<r<-6 \mathrm{~mm}$ ) in Fig. 7c becomes larger than those in Fig. 7d. It should be noted that the flame fluctuates at the shear layer, which is expected to show high levels of fluctuation regardless of the presence of the flame. Thus, the local quenching could amplify the apparent velocity fluctuation.
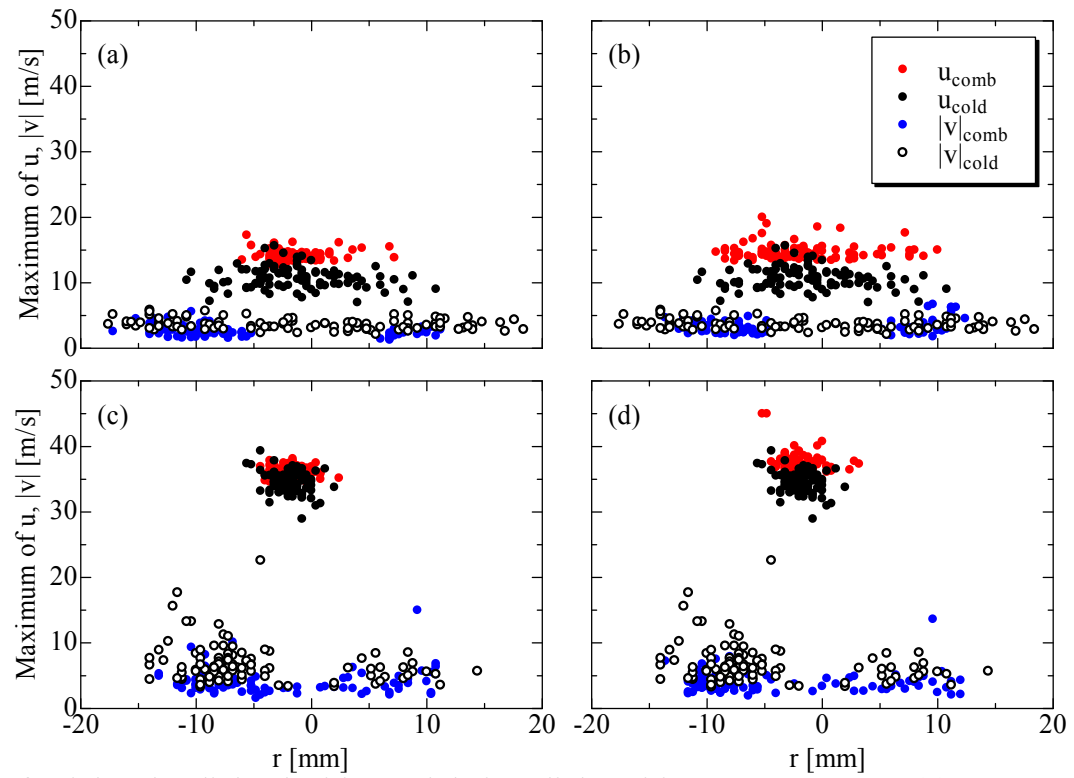

Fig. 6 Maximum of axial and radial velocities and their radial positions at $z=15 \mathrm{~mm}$; (a) $U_{m}=10 \mathrm{~m} / \mathrm{s}, \phi_{m}=0.75$, (b) $U_{m}=$ $10 \mathrm{~m} / \mathrm{s}, \phi_{m}=0.90$, (c) $U_{m}=30 \mathrm{~m} / \mathrm{s}, \phi_{m}=0.75$ and (d) $U_{m}=30 \mathrm{~m} / \mathrm{s}, \phi_{m}=0.90$. To examine the effect of the flames, the measurements of cold flow without flames were also conducted.
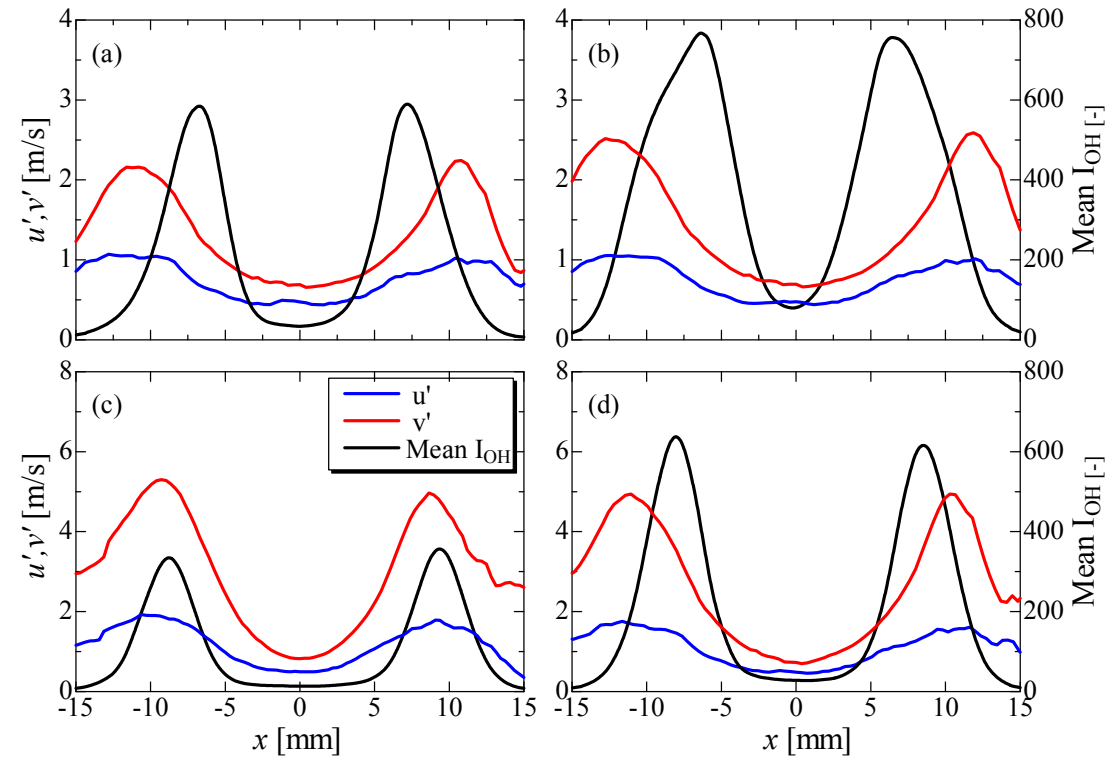

Fig. 7 Radial distributions of rms axial, radial velocity and mean $\mathrm{OH}$ fluorescence signal obtained at $z=15 \mathrm{~mm}$; (a) $U_{m}=$ $10 \mathrm{~m} / \mathrm{s}, \phi_{m}=0.75$, (b) $U_{m}=10 \mathrm{~m} / \mathrm{s}, \phi_{m}=0.90$, (c) $U_{m}=30 \mathrm{~m} / \mathrm{s}, \phi_{m}=0.75$ and (d) $U_{m}=30 \mathrm{~m} / \mathrm{s}, \phi_{m}=0.90$. 
Filatyev et al. (2005) have pointed out that the reaction of the flame zone is affected by the stretch rate, which is strongly related with the velocity gradient. Hence, it is considered that the above local velocity fluctuation could cause the local extinction. In our recent measurement, we evaluated the strain rate of turbulent flames in the cyclone-jet combustor [Yamamoto et al., 2016]. The strain rate was obtained by the following equation.

$$
\kappa_{s}=\frac{\partial u_{t}}{\partial s}
$$

where $s$ is the coordinate along the flame front, and $u_{t}$ is the velocity component that is tangential to the flame surface [Driscol et al., 1994]. Similarly, we obtained the value of $\kappa_{\mathrm{s}}$ in this study. Needless to say, it cannot be evaluated when the flame front disappears. Then, the local strain rate along the flame was calculated only when the length of the continuous flame front was longer than $2 \mathrm{~mm}$. Results are shown in Fig. 8. It should be noted that the local flame extinction was only observed for $U_{m}=30 \mathrm{~m} / \mathrm{s}$ and $\phi_{m}=0.75$. As seen in this figure, there are several interesting findings. First, when the equivalence ratio is larger, the higher strain rate is observed. However, independent of the mean exit velocity or the equivalence ratio, the minimum value of the strain rate is almost the same. On the other hand, as for the maximum value, the large difference is observed for each condition. In the case of $U_{m}=30 \mathrm{~m} / \mathrm{s}$, for $\phi_{m}=$ 0.75 , the value over $700 \mathrm{~s}^{-1}$ is not seen. On the other hand, for $\phi_{m}=0.90$, the flame is formed even at the higher strain rate. Therefore, it is expected that the flame could not be maintained at $\kappa_{\mathrm{s}}>700 \mathrm{~s}^{-1}$. Although more measurements may be needed for further discussion, it can be concluded that the high strain rate could cause the local flame extinction through the strong velocity fluctuation.

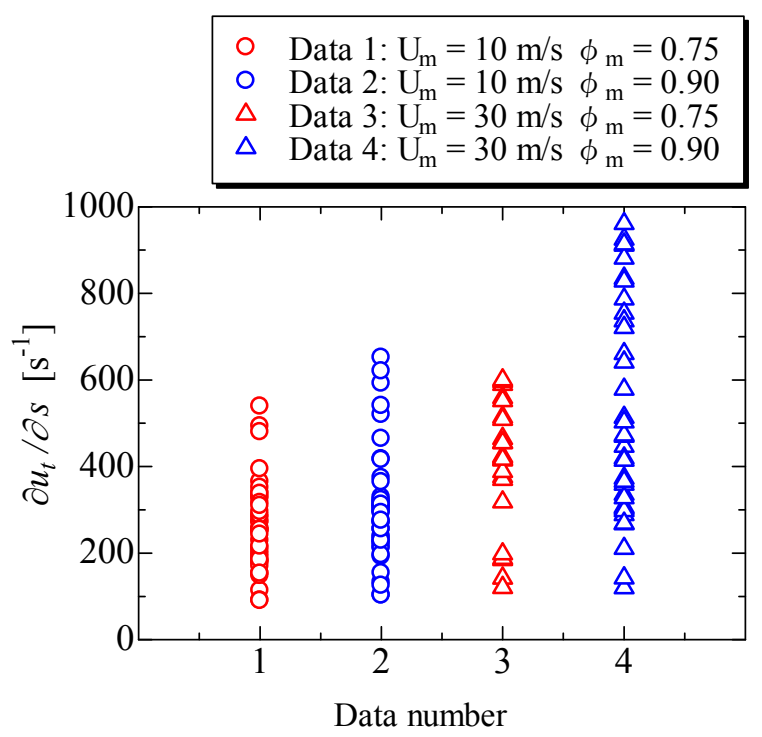

Fig. 8 The measured strain rate along the flame front by simultaneous PIV/OH-PLIF.

\subsection{Flame surface density}

Finally, the flame surface density (FSD) was discussed, corresponding to the expectation of the flame surface area per unit volume. We used the orthogonal OH-PLIF in vertical and horizontal planes to collect 1000 images. We evaluated the flame surface density based on 2D and 3D coordinates, simply describing $\Sigma_{2}$ and $\Sigma_{3}$ [Hawkes et al., 2011]. The flame front was firstly determined by the $\mathrm{OH}$ image [Yamamoto et al., 2011]. Then, the flame surface density was obtained:

$$
\Sigma_{3}=\frac{1}{V_{f}} \int L(z) d z
$$

where $V_{f}$ is the volume of flame brush, and $L(z)$ is the flame perimeter at the axial coordinate (at any horizontal plane). For the flame perimeter, the circular flame length was calculated based on the axisymmetry. To consider the flame wrinkling of the actual flame, the increasing ratio of $R$ must be multiplied, which is the ratio of the real flame perimeter to the circle length of mean flame radius. Eq. 2 can be written as follows: 


$$
\Sigma_{3}=\frac{1}{V_{f}} \int R \times(2 \pi r(z)) d z
$$

where the radial position of the flame front is $r(z)$. That is, the complex flame perimeter can be counted by the asymmetrical assumption corrected by the concave and convex flame shape with $R$. It has been found that $R$ is independent of $z$. The volume of flame brush, $V_{f}$, is the region where the flame front is detected. On the other hand, 2D FSD $\left(\Sigma_{2}\right)$ was evaluated based on the vertical plane, corresponding to the flame length density per unit flame area.

Results are shown in Fig. 9. It is seen that, for 2D and 3D cases, the flame surface density increases with an increase in $U_{m}$, but $\Sigma_{3}$ is much larger than $\Sigma_{2}$ for $U_{m}>20 \mathrm{~m} / \mathrm{s}$. The range of 3D FSD is from 0.2 to $0.8 \mathrm{~mm}^{-1}$, which is similar to the reported data of the Bunsen flame [Filatyev et al., 2005]. As the equivalence ratio is higher, FSD becomes smaller. Hawkes et al. (2011) have reported that $\Sigma_{3}$ is larger than $\Sigma_{2}$ by $4 / \pi$ in the case of homogeneous turbulence. In our case, as seen in Fig. 7, the rms axial and radial velocities are not the same. Thus, the increasing factor of 3D effects may be different. Another probable explanation is that the value of $4 / \pi$ may not be suitable for our axisymmetric burner, because it was computed in a Cartesian geometry. This could be why the increase of 2D FSD with $U_{m}$ is small. That is, as the bulk velocity increases, the flame radius increases as well as the fluctuations. Portions of flame length at a small radius will thus have a really small weight in the real flame surface density compared to portions of flame length at a larger radius.

For further study, the variation of 3D FSD with the rms axial velocity was examined. Results are shown in Fig. 10. To compensate the burning velocity at different equivalence ratio, the rms axial velocity was non-dimensionalized by the laminar burning velocity of $S_{L 0}$. Here, $u$ ' is the value of unburned gas at the center axis (see Fig. 7). A good correlation between $u^{\prime}$ and $\Sigma_{3}$ is observed. Hence, it can be derived that the increase of FSD is simply caused by the flame wrinkling due to the turbulence.

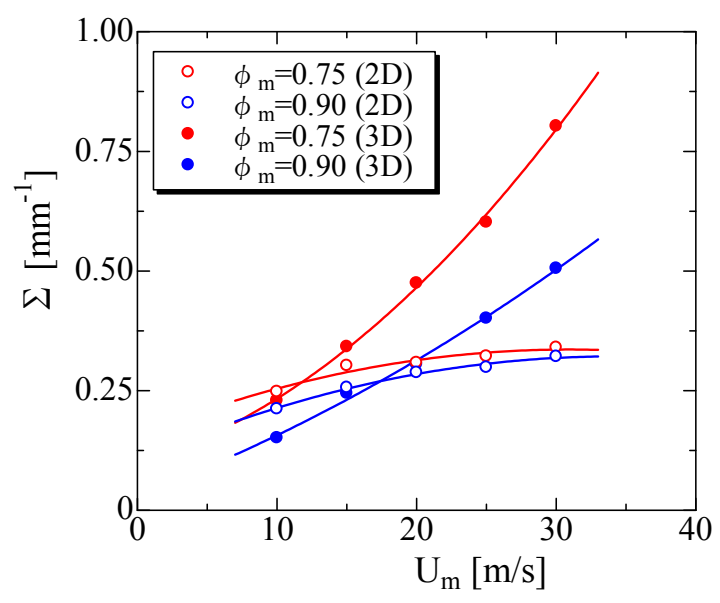

Fig. 9 Flame surface density in 2D and 3D.

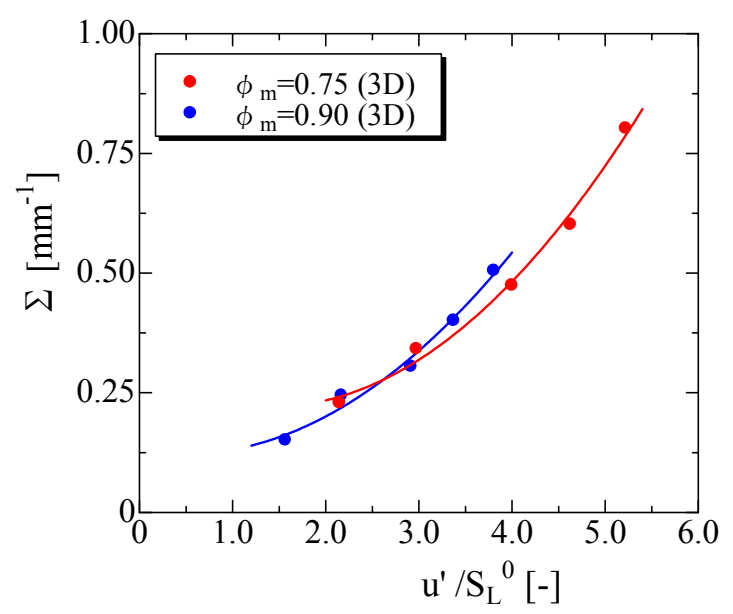

Fig. 10 Variation of flame surface density with rms axial velocity. 
Here, the integrated flame surface density was also calculated. It was the total flame surface area based on 2D and 3D imaging. Results are shown in Fig. 11, obtained by a direct measurement of the flame surface area based on the $\mathrm{OH}$ images. The same approach [Yamamoto et al., 2011] was adopted. The values of 2D and 3D were compared. Interestingly, the variation of the integrated value with $u^{\prime} / S_{L 0}$ is quite linear. This implies that the flame surface area is always increased due to the turbulence, even though the local extinction occurs. The integrated $2 \mathrm{D}$ flame surface density is much smaller than that of 3D. The increasing factor of 3D effects is about 1.5 to 2.3 , which is similar to that of the Bunsen flame [Chen, 2009].

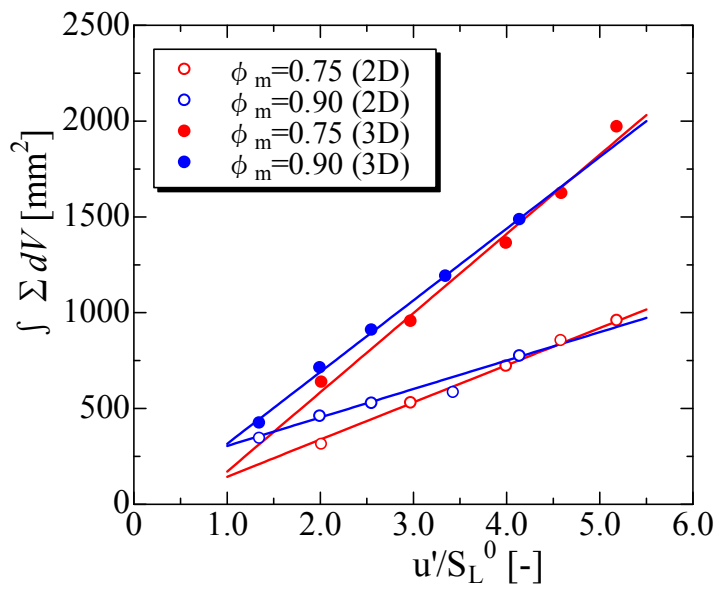

Fig. 11 Integrated flame surface density in 2D and 3D.

\section{Conclusions}

The simultaneous PIV/OH-PLIF measurements were conducted for propane turbulent premixed flames. The following conclusions were derived.

(1) In the cyclone-jet combustor, the axial velocity becomes the maximum around the center axis, whereas the rms axial or radial velocity takes its maximum at the position far from the center axis. A good correlation between axial and radial rms velocities is observed. The larger velocity fluctuation is observed by increasing the mean exit velocity of $U_{m}$. However, the radial position of the peak $\mathrm{OH}$ fluorescence signal is not in the region of the large rms velocity, except for the condition of $U_{m}=30 \mathrm{~m} / \mathrm{s}$ and $\phi_{m}=0.75$ where the local extinction is observed. Interestingly, at the condition where the local extinction occurs, the high strain rate over $700 \mathrm{~s}^{-1}$ is not seen. Then, it is considered that the large velocity fluctuation induces the local extinction through the high strain rate.

(2) The flame surface density is larger, as $U_{m}$ is increased. The range of 3D FSD is from 0.2 to $0.8 \mathrm{~mm}^{-1}$, which is similar to that of the Bunsen flame. Based on FSDs estimated by the 2D and 3D imaging, the increasing factor of $3 \mathrm{D}$ effects is not the same value of homogeneous turbulence. Recognizing a linear relationship between rms axial velocity and integrated FSD, the increase of FSD is simply caused by the flame wrinkling due to the turbulence, even though the local extinction occurs.

\section{References}

Avallone G., Gregorio, F.D. and Romano, G.P., PIV measurements in grid turbulence, Proceedings of 5th International Symposium on Particle Image Velocimetry (2003), pp. 22-24.

Bradley, D., Haq, M.Z., Hicks, R.A., Kitagawa, T., Lawes, M., Sheppard, C.G.W. and Woolley, R., Turbulent burning velocity, burned gas distribution, and associated flame surface definition, Combustion and Flame, Vol. 133 (2003), pp. 415-430.

Cant, R.S., Pope, S.B. and Bray K.N.C., Modelling of flamelet surface to volume ratio in turbulent premixed combustion, Proceedings of the Combustion Institute, Vol. 23 (1990), pp. 809-815.

Chen, Y.C., Measurements of three-dimensional mean flame surface area ratio in turbulent premixed Bunsen flames, Proceedings of the Combustion Institute, Vol. 32 (2009), pp. 1771-1777. 
Driscoll, J.F., Turbulent premixed combustion: Flamelet structure and its effect on turbulent burning velocities, Progress in Energy and Combustion Science, Vol. 32 (2008), pp. 91-134.

Driscoll, J.F., Sutkus, D.J., Roberts, WM.L., Post, M.E. and Goss, L.P., The strain exerted by a vortex on a flame determined from velocity field images, Combustion Science and Technology, Vol. 96 (1994), pp. 213-229.

Filatyev, S.A., Driscoll, J.F., Carter, C.D. and Donbar, J.M., Measured properties of turbulent premixed flames for model assessment, including burning velocities, stretch rates, and surface densities, Combustion and Flame, Vol. 141 (2005), pp. 1-21.

Gülder, Ö.L., Contribution of small scale turbulence to burning velocity of flamelets in the thin reaction zone regime, Proceedings of the Combustion Institute, Vol. 31 (2007), pp. 1369-1375.

Hawkes, E.R., Sankaran, R. and Chen J.H., Proceedings of the Combustion Institute, Vol. 33 (2011), pp. 1447-1454.

Jainski, C., Rißmann, M., Böhm, B. and Dreizler, A., Experimental investigation of flame surface density and mean reaction rate during flame-wall interaction, Proceedings of the Combustion Institute, Vol. 36 (2017), pp. 1827-1834.

Kido, H., Nakahara, M., Nakashima, K. and Hashimoto, J., Influence of local flame displacement velocity on turbulent burning velocity, Proceedings of the Combustion Institute, Vol.29 (2002), pp. 1855-1861.

Nishiki, S., Hasegawa, T., Borghi, R. and Himeno, R., Modeling of flame-generated turbulence based on direct numerical simulation databases, Proceedings of the Combustion Institute, Vol. 29 (2002), pp. 2017-2022.

Osborne, J.R., Ramji, S.A., Carter, C.D. and Steinberg, A.M., Relationship between local reaction rate and flame structure in turbulent premixed flames from simultaneous $10 \mathrm{kHz}$ TPIV, OH PLIF, and CH2O PLIF, Proceedings of the Combustion Institute, Vol. 36 (2017), pp. 1835-1841.

Peters, N., Laminar flamelet concepts in turbulent combustion, Proceedings of the Combustion Institute, Vol. 21 (1986), pp. 1231-1250.

Peters, N., The turbulent burning velocity for large-scale and small-scale turbulence, Journal of Fluid Mechanics, Vol. 384 (1999), pp. 107-132.

Suzuki, H. and Yamamoto, K., Effect of swirl flow on flame structure and stability in turbulent combustion, Transactions of the Japan Society of Mechanical Engineers, Series B, Vol. 79, No. 804 (2013), pp. $319-328$ (in Japanese).

Yamamoto, K., Inoue, S., Yamashita, H., Shimokuri, D., Ishizuka, S. and Onuma, Y. PIV measurement and turbulence scale in turbulent combustion, Heat Transfer Asian Research, Vol.35 (2006), pp.501-512.

Yamamoto, K., Inoue, S., Yamashita, H., Shimokuri, D. and Ishizuka, S., Flow field of turbulent premixed combustion in a cyclone-jet combustor, Journal of Thermal Science and Technology, Vol. 2, No. 1 (2007), pp. 90-101.

Yamamoto, K., Ishii, S. and Ohnishi, M. Local flame structure and turbulent burning velocity by joint PLIF imaging, Proceedings of the Combustion Institute, Vol. 33 (2011), pp. 1285-1292.

Yamamoto, K., Nishizawa, Y. and Onuma, Y., Persistence of laminar flamelet structure under highly turbulent premixed combustion, JSME International Journal, Series B, Vol. 47, No. 6 (2003), pp. 408-415.

Yamamoto, K. Ohnishi, M., Hayashi, N., Ozeki, M. and Yamashita, H., Flame image and flame structure of turbulent premixed flames using simultaneous OH-HCHO PLIF technique, Transactions of the Japan Society of Mechanical Engineers, Series B, Vol. 73, No. 733 (2007), pp. 1943-1949 (in Japanese).

Yamamoto, K., Ozeki, M., Hayashi, N. and Yamashita, H., Burning velocity and OH concentration in premixed combustion, Proceedings of the Combustion Institute, Vol. 32 (2009), pp. 1227-1235.

Yamamoto, K. Yamamori, K. and Hayashi, N., Simultaneous measurements of flame geometry and flow field by OH-PLIF/Stereo PIV, Transactions of the Japan Society of Mechanical Engineers, Series B, Vol. 82, No. 844 (2016), pp. 1-15 (in Japanese).

Wabel, T.M., Skiba, A.W., Temme, J.E. and Driscoll, J.F., Measurements to determine the regimes of premixed flames in extreme turbulence, Proceedings of the Combustion Institute, Vol. 36 (2017), pp. 1809-1816. 